\title{
Telemedicine for Women's Health During COVID-19 Pandemic in India: A Short Commentary and Important Practice Points for Obstetricians and Gynaecologists
}

\author{
Vimee Bindra ${ }^{1}$
}

Received: 16 June 2020 / Accepted: 26 June 2020 / Published online: 16 July 2020

(c) Federation of Obstetric \& Gynecological Societies of India 2020

\begin{abstract}
Background/purpose of study In view of restrictions on patients because of COVID-19 pandemic, face-to-face consultations are difficult. This short commentary tells us about the feasibility of telemedicine in this scenario in obstetrics and gynaecology.

Methods The database from our teleconsultation application (Apollo 247 and Askapollo) was analysed to assess feasibility of telemedicine and to design a triage pathway to reduce hospital visits for non-emergency situations and also to identify emergency cases without delay during this lockdown phase. Existing guidelines by Ministry of Health and Family Welfare (MOHFW), Government of India, were accessed.

Results This was a single-doctor experience of 375 consultations done over 65 days. We also designed a triage pathway for obstetrics and gynaecology cases, and we discussed general practice for obstetricians and gynaecologists with its utility and limitations.

Conclusion Telemedicine has provided us the opportunity to manage women health problems and pregnancy concerns during this pandemic of COVID-19, except a few instances where face-to-face consultation or hospital visit is must. If we implement the triage pathway, we can minimize the risk of exposure for both patients and healthcare teams during COVID-19 pandemic.
\end{abstract}

Keywords Telemedicine $\cdot$ Obstetrics and gynaecology $\cdot$ Pandemic $\cdot$ Lockdown

\section{Introduction}

Disasters and pandemics pose a great challenge to health care delivery for an already burdened healthcare system. When the whole world is fighting with an invisible enemy, there is a major shift in routine patient care. WHO declared COVID 19 as pandemic on March 11, 2020 [1]. Many hospitals and practices had to cancel routine out-patients visits and out of necessity, most of the practices have been encouraged to use telemedicine as a method of continuity of care.

While writing this article, there were already 7,731,721 cases globally and 4,28,210 deaths [1]. India had reported

Dr. Vimee Bindra MS (Gynaecology and Obstetrics) is a consultant gynaecologist and obstetrician at Apollo Hospitals, Hyderabad, India.

Vimee Bindra

vimee.bindra@gmail.com

1 Apollo Hospitals, Hyderabad, India
309,603 cases and 8890 deaths [2]. Pregnant women are the vulnerable population hence guidance and support through telemedicine will go a long way in reducing complications and timely intervention during this pandemic.

Known consequences of delayed access to healthcare due to lockdown and pandemic situation on pregnant women could be delay in identifying the warning signs, more maternal and neonatal deaths, less access to abortion facilities as patients are also scared to visit hospitals because of fear of contracting the infection. During these times telemedicine came as a boon for our patients when Govt of India and Medical Council of India released their new guidelines for use of telemedicine during this pandemic [3].

Our hospital telemedicine services came to our rescue and through our applications Apollo 247 and Ask Apollo we could serve a large number of women in need during this pandemic situation.

A total of 375 tele consults (single doctor experience) happened during 25th March to 31st May. The age range of patients who consulted via telemedicine were 18-60 years. 
$87 \%$ of patient who consulted through telemedicine were from a nearby location and $13 \%$ were outstation from remote locations or cities where nearby clinics and outpatient facilities were closed. The new patients who consulted for the first time constituted $27.5 \%$ of total consults, while follow up patients constituted $72.5 \%$ of the tele consults. (New patients mean they did not have any face to face consultation in the past 6 months and follow up patient had at least one face to face consultation in the past 6 months.)

Out of 375 consultations, $66 \%$ consultations happened for one time, $20 \%$ for two times, and rest of the consultation that is $14 \%$ happened for three or more than three times. Multiple consultations for same patients were mostly for pregnant patients.

As we know significant number of COVID-19 infections are caused by asymptomatic carriers, decreasing in person contact with patients is of vital importance [4]. The described triage pathway (Figs. 1,2) for teleconsultation allows for necessary urgent and emergent obstetric or gynaecologic care and helps in minimizing the exposure that would be associated with standard obstetric and gynaecology consultations. Many specialties like orthopaedic and urology are using telemedicine extensively $[5,6]$.

Before COVID-19 pandemic, telemedicine had not been widely used by Indian physicians. It is there for distant telehealth services where rural areas are connected to hospitals through telemedicine.

\section{Telemedicine and Women's Health}

Women's health especially pregnant women can utilize this facility and avoid contracting the infection. For gynaecological disorders if no emergency can also be managed through telemedicine from the comfort of their homes. Gynaecologists and obstetricians can interact with patients, gather complete history, educate them regarding the warning signs in pregnancy and give advice and instruct them to come for physical consultation if needed. Telemedicine in India can go a long way in future at least for non-emergencies and low risk pregnancies. Also, not only covid-19, this can help to reduce transmission of many diseases and reduce overall disease burden on healthcare.

The following points can be useful practical guide for telemedicine for registered medical practitioners. Few of these have been adapted from Ministry of Health and Family Welfare (MOHFW) [3].

Consent is mandatory.

General considerations

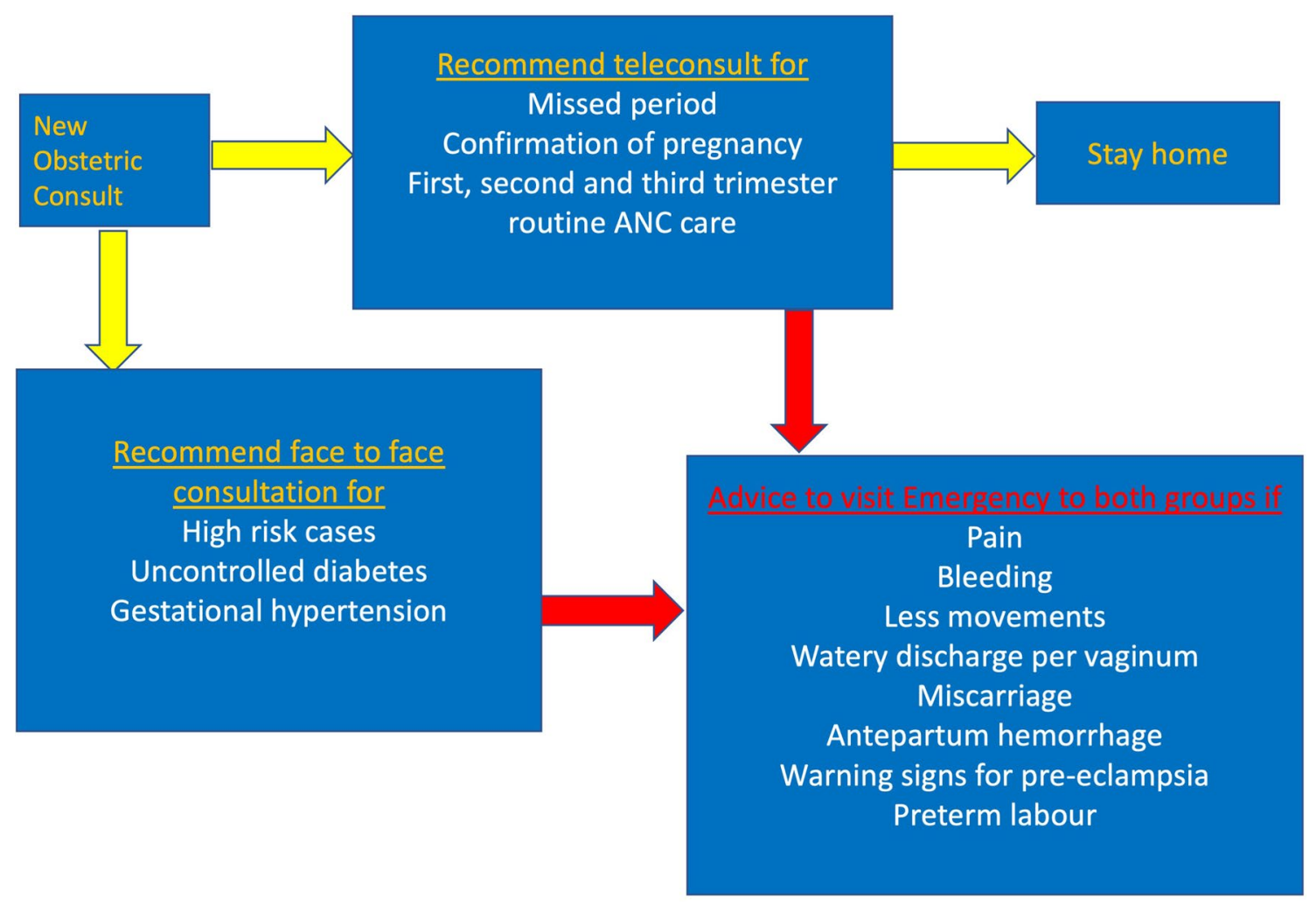

Fig. 1 Obstetrics consultation triage 


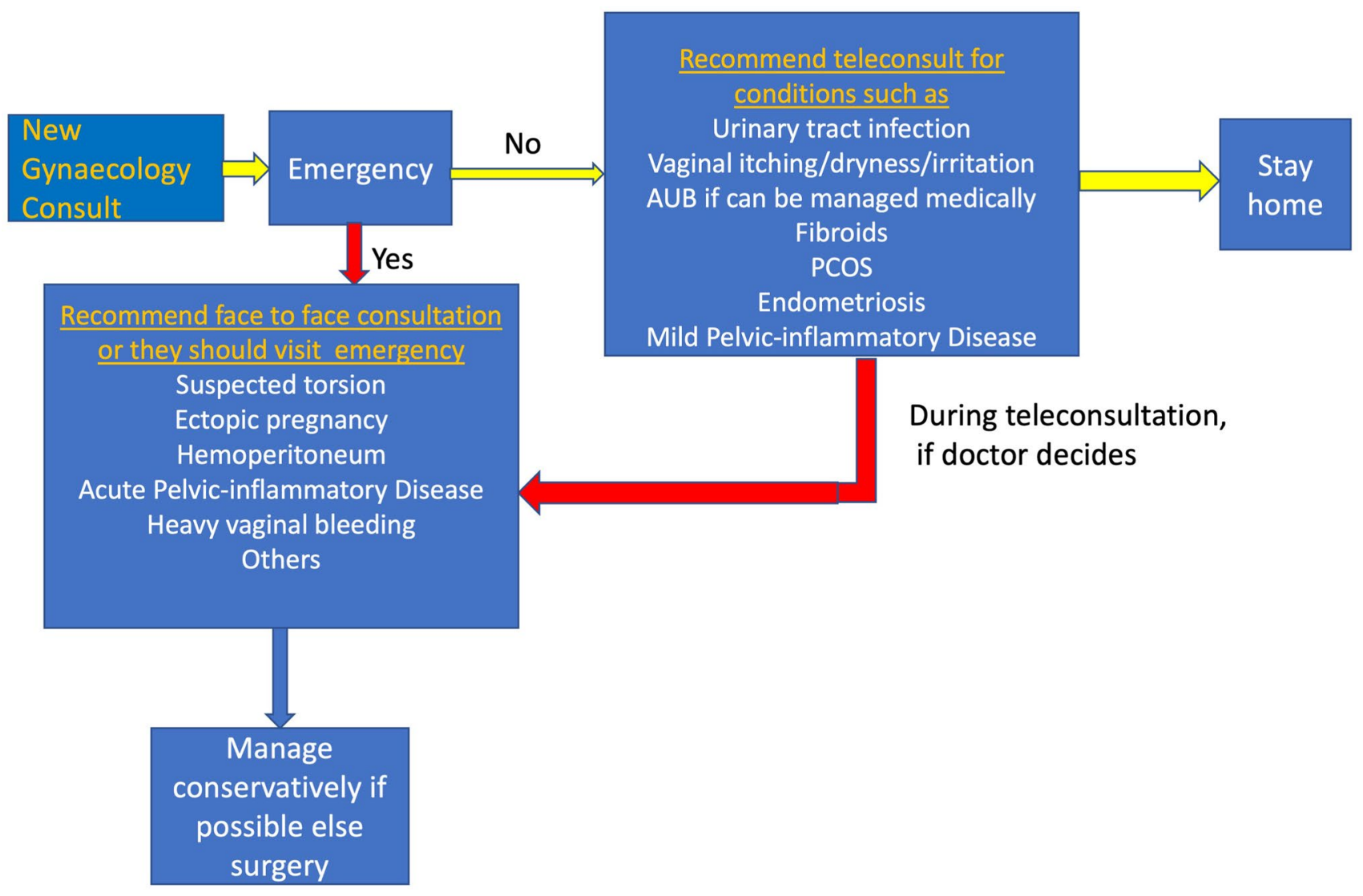

Fig. 2 Gynaecology consultation triage

- Maintain confidentiality, medical record with reports, laboratory investigations and prescription.

- Patient can be charged for consultation.

- Schedule X prescription drug, narcotics or psychotropic substances cannot be prescribed.

\section{History}

- Complete history/presenting complaints.

- Old record if any, scans and blood reports.

- Rule out allergies.

- Assessment of her understanding of self-care/education regarding fetal movements/warning signs.

Examination

- Patient and doctor both should understand that telemedicine is not a substitute for physical examination.

- If required patient should be called for face to face consultation immediately (Figs. 1, 2).

Prescription

- Healthy lifestyle education.
- Pregnant patients in second and third trimester should be taught about "daily fetal movement count", signs of labour, and identifying leaking.

- Along with general gynaecological and obstetric advice, they should be taught about COVID-19 situation and importance of hand washing, sneezing etiquettes, social distancing should be explained.

New cases

- Any first consult or follow up more than 6 months, video consultation is preferable.

- New prescription issued should have all the information such as name, age, complaints, clearly written medications, doctor's registration number and medications should be explained well with the time of intake as well as before or after food.

Special situation requiring face to face consultation

- Pregnant patients with missed abortion, with labour pains, PROM, gestational hypertension, GDM (if uncontrolled), ante-partum haemorrhage, ectopic pregnancy, molar pregnancy. 
- For gynaecological patients with heavy menstrual bleeding not responding to medication, suspected ovarian torsion, acute pelvic inflammatory disease and others.

By utilizing telemedicine for non-urgent gynaecologic and obstetric consultations, we were able to provide appropriate care and counselling, while reducing the surge of outpatient gynaecologic and obstetric visits and care following COVID-19 crisis.

\section{Conclusion}

Telemedicine has provided us the opportunity to manage women health problems and pregnancy concerns during this pandemic of COVID-19, except a few instances where face to face consultation or hospital visit is must. If we implement the triage pathway we can while minimize the risk of exposure for both patients and healthcare teams during COVID-19 pandemic. More robust data is needed to evaluate the effectiveness of telemedicine to manage antenatal women and general gynaecological issues in India and this can be utilized in future too for continuity of care.

Acknowledgements I express my thanks and acknowledge Mr. Atulya (Apollo $247 \mathrm{app}$ ) and Mr. Bhaskar (Ask Apollo) for providing me with the data from app for analysis.

\section{Compliance with ethical standards}

Conflict of interest The author declares that there is no conflict of interest

Ethical statement This article does not contain any studies with human participants by any of the authors.

Informed consent All patients who were consulted through telemedicine had consented to teleconsultation and they understood the nature of consultation as compared to physical consultation.

\section{References}

1. World Health Organization. Coronavirus disease (COVID-19) pandemic. https://www.who.int/emergencies/diseases/novel-coron avirus-2019. Accessed 5 May 2020.
2. Ministry of Health and Family Welfare Govt. of India. https:// www.mohfw.gov.in/. Accessed 5 May 2020.

3. https://www.mohfw.gov.in/pdf/Telemedicine.pdf. Accessed 5 May 2020.

4. Li R, Pei S, Chen B, et al. Substantial undocumented infection facilitates the rapid dissemination of novel coronavirus (SARSCoV2). Science. 2020. https://doi.org/10.1126/science.abb3221.

5. Tanaka MJ, Oh LS, Martin SD, Berkson EM. Telemedicine in the era of COVID-19: the virtual orthopaedic examination. J Bone Joint Surg Am. 2020. https://doi.org/10.2106/JBJS.20.00609.

6. Borchert A, Baumgarten L, Dalela D, et al. Managing urology consultations during COVID-19 pandemic: application of a structured care pathway. Urology. 2020. https://doi.org/10.1016/j.urolo gy.2020.04.059.

Publisher's Note Springer Nature remains neutral with regard to jurisdictional claims in published maps and institutional affiliations.

\section{About the Author}

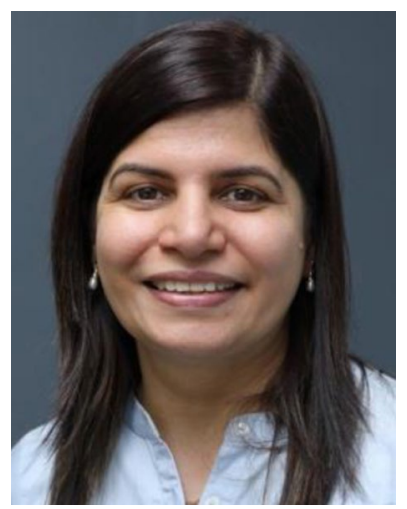

Dr. Vimee Bindra completed her basic medical education from $\mathrm{R}$ G Kar Medical College, Kolkata (1998-2003) and post-graduation in Obstetrics and Gynecology Kolkata (2005-2008), followed by a Diploma in Advanced Gynecological Endoscopy from BEAMS Hopsitals, MUMBAI and advanced Laparoscopy and Hysteroscopy training from Clermont Ferrand (FRANCE). She has a keen interest in the field of Gynecological Endoscopy specially Endometriosis Excision Surgeries and Fertility enhancing surgeries. She has received her Advanced Reproductive Technology (ART) Training from, Homerton University Hospital, London (UK). She has done her Fellowship in Cosmetic Gynaecology from American Aesthetic Association. She was awarded "Vaidya Sree Award in 2019" and "Best Emerging Gynaecologist of the year" in 2020. She is an author of three well known medical books in the field of Gynaecology and Obstetrics. 